\title{
Patient Satisfaction And Low OPD At Dental Colleges: Review Literature
}

\author{
Priyanka Bhushan ${ }^{1}$, Tanya Nandkeoliar ${ }^{2}$, Rohit Agarwal ${ }^{3}$, Kundan Kumar $^{4}$ \\ ${ }^{I}$ (Dept of Public Health Dentistry, RIMS Dental College, Imphal, India) \\ ${ }^{2}$ (Dept of Periodontology, RIMS Dental College, Imphal India) \\ ${ }^{3}$ (Dept of Public Health Dentistry, Maitri College of Dentistry \& Research Centre, Durg, India) \\ ${ }_{4}^{4}$ (Army Dental Corps, India)
}

\begin{abstract}
Health is one of the most challenging sectors in the underdeveloped and developing countries. In countries like India where majority of population do not have access to medical health; oral health is of least importance. Prevalence of dental diseases has been steadily increasing over the years especially oral cancerous lesions. However despite the improved dentist to population ratio and substantial increase in number of dental college we are yet to achieve the desired oral health status as the problem lies not in number but on the distribution of college and dental surgeon. Increased appointment time and OPD procedure followed by dental college were cited as the major reason for low OPD at Dental Colleges. There is urgent need to bring about certain changes in the oral health programme and OPD procedure followed by the dental colleges and hospital. Use of Information Technology to streamline the OPD procedure should be implemented. Effort should be made to make the dental services as accessible to the general population as possible. Tax incentives and benefits for dentist and dental hospital opened at rural area should be thought of. Dental post at every PHC's should be created so as to make dental treatment accessible to population along with medical facilities.
\end{abstract}

Keywords: Dental College, Health, OPD, Satisfaction

\section{Introduction}

Health condition of the population of underdeveloped countries has always been a matter of grave concern. In majority of the underdeveloped countries health sector is very low on the priority list of the government agenda. Government of developing countries like India is always under dilemma to choose between economic growth and social sector. In developing countries like India where vast majority of population do not have access to medical health, portable water, satisfactory hygiene and sanitation etc oral health is of least importance. Together with this increased socio-economic divide, high dental treatment cost, negligible government aided dental hospitals, poor oral habits like tobacco, betel nut \& paan chewing etc and food habits leads to poor oral hygiene status of the population. In a vast and developing country like India, it is necessary to involve private sector, NGO's and PPP model to achieve the desired health care goal.

DCI's National survey was conducted to determine prevalence of dental diseases in Indian states in 2002-03 (1). The survey reports that prevalence of most common dental disease i.e. dental caries is in the range of 50-84.7\% distributed among various age groups and that of periodontal disease is in the range of $66.2-89.2 \%$. Oral cancers have a prevalence rate of $0.2-0.4 \%$. About $24 \%$ of the populations are into the habit of tobacco chewing \& smoking that can predispose to number of dental as well as many systemic diseases (1). Prevalence of dental diseases has been steadily increasing over the years especially oral cancerous lesions. Ferlay et al. in 2013 reported that the incidence of oral cancers among Indians is 7.6\%, and mortality due to the same is $7.6 \%$ annually signifying the need for immediate action (2).

WHO recommends dentist to population ratio of 1:7500. In 1960's dentist to population ratio in India was 1:300,000(3). To overcome this discrepancy and to make dental treatment more accessible the Dental Council India (regulatory body of dental education in India) granted private entities and Trust to start dental colleges in India (4). The result is that at present there are about 294 dental colleges producing more than 30,000 graduates every year and the dentist to population ratio in India now stands at 1:10,000 (3), although still below WHO standards. However despite this improvement we are yet to achieve the desired oral health status as problem lies not in number but on the distribution of college and dental surgeon. Majority of the dentist (80\%) are working in big cities despite the fact that larger proportion of Indian population (70\%) still resides in rural areas (3). In order to be economically viable and attract large number of students to their college, most of the colleges were opened in the urban areas or their outskirts (4). 


\section{Reasons For Low OPD At Dental College}

Despite the presence of large number of dental college in various states of India the OPD stats of colleges are disappointing. Various studies have been conducted to ascertain the reasons for low OPD and dissatisfaction among patients at various Dental College. Some of the most prominent reasons for low OPD and dissatisfaction are as follows:

\subsection{Waiting Time/Appointment Time}

Patients' waiting time has been defined as "the length of time from when the patient entered the outpatient clinic to the time the patient actually leaves the OPD" (5). Waiting time was considered to be the most important factor for patient satisfaction $(6,7)$.OPD of a hospital is the first point of contact between patient and hospital and should be considered as the most important factor in patient satisfaction.

In a study conducted by Kashinath KR et al $60 \%$ of the respondent said that waiting time for treatment affected their satisfactory level while attending the OPD of a dental college in Tumkur (8). Similar views were reported by $43.66 \%$ of the patient and $28 \%$ of the faculty staff of a dental college in study conducted by Bhushan P et al (4) whereas $38 \%$ and $27 \%$ of the respondent wanted improvement in the registration process and waiting time in a study conducted by Bither S \& Gandhi S at a dental college in Ludhiana, Punjab (9). Majority of the dental college \& hospital are still using old and primitive method of hand written data entry and maintaining of register at registration counter and at the reception of every department. Use of computer and peripherals are negligible thereby leading to loss of valuable time and dissatisfaction among the patients.

\section{Use of Information Technology in OPD}

Use of IT at OPD of any dental college is negligible and the authorities are still using manual data entry at reception and department. It is important to have hard copy of OPD slip along with treatment card but the same data should also be digitalized so that it is easy to retrieve the patient records. Cases of clinical importance are often delayed due to discussions between students and teachers.

The college authority should provide stand alone PC's for each and every department from reception to clinical department to store, radiology, lab etc and all the computers should be connected to each other with the help of LAN (Local Area Network). There are specially designed software (10) ex (Densoft Software Fig 1) were in the details of the patient can be entered at one place and that detail can be assessed by any department and concerned authority where computer is available and connected with LAN. At the concerned department the treatment provided can be entered and digitally stored and retrieved when required.

\section{Electronic Health Record (EHR) (11)}

EHR was originally envisioned as an electronic file cabinet for patient data from various sources (integrated text, voice, images, hand written notes etc). This includes automated order entry and patient tracking system providing real time assess to patient data, as well as a continuous longitudinal record of their care. In case the patient loses its health card then his/her records can be easily retrieved leading to lot of savings in time both for the doctor as well as patient. Also these data \& record can be utilized for academic purpose and can be easily shared with other institute or doctors for further specialized advice (11).

\section{Picture Archiving \& Communication System (PACS)}

This technology- captures and integrates diagnostic and radiological images from various devices (for example X-ray, MRI, CT scan), Stores them; disseminate them to a medical record, a clinical data repository or other points of care (11). PACS can lower cost for acquiring and storing films by storing digitized radiology images, and may reduce the workload among the radiology staff (12).

This will also help in reducing patient treatment time as the radiographs can be digitally transferred to the concerned department or shared on the LAN computer and can be viewed by the doctor at any department. Also the clinical features can be digitally recorded and these can be utilized for clinical discussions after the patient treatment has been completed.

\subsection{OPD Layout \& Procedure}

Within a dental college there are approximately 9 fully functional departments. In private dental clinic various dental treatment procedures are available under one roof, unlike a dental college where there are specific departments for specific treatment. Patient complains of difficulty in locating the departments and lengthy OPD procedures as the reason for dissatisfaction with dental college OPD. In a study conducted by Asghar S et al $23.7 \%$ of the respondents suggested that there should be more guiding sign boards in finding respective sections at Bahria Dental College Hospital, Karachi (13). Similar views were represented by $81 \%$ of the respondent in a study conducted at Tumkur dental college by Kashinath KR et al (8). $29.66 \%$ of the respondent were of view that lot of time was wasted in locating the department and also in lengthy OPD procedure in a study conducted 
by Bhushan P et al (4). In most of the dental college's administrative block, academic section, labs and clinical departments are clubbed together in same building. The location of the clinical department of the hospitals are not according to the utilization pattern of the dental services but rather depends on the space available with the college authorities. Together with this the complex building structure and absence of proper sign board and maps reflecting the department's location leads to loss of valuable time and difficulty for the patients.

\section{Reorganization of OPD\& Procedure}

The most frequently used departments in a dental college are the Oral Surgery, Prosthodontics, Periodontology, Endodontic and Radiology department. In a study conducted by Abdurrazaq $\mathrm{T}$ et al at Federal Medical Centre, Katsina, Northwest Nigeria restorative and surgical procedures were the major treatment utilized $(40.5 \%)$ and $(40 \%)$ respectively by the patients while periodontal procedure were utilized by $(18.6 \%)$ patients (14). In a study conducted by Vashisth S et al, on utilization of dental services in rural area of Haryana oral prophylaxis $(51.2 \%)$, restorations $(22.9 \%)$ and extractions $(8.8 \%)$ were the major treatment utilized (15).

However in majority of the dental college OPD layout depends upon the space available. It is important to have the most widely used department at ground floor so that it is easily accessible and convenient for the patient to locate them. The proximity of these departments with reception, payment counter and radiology \& diagnostic department will also save lot of time for patients. "The OPD should be on the ground floor near diagnostic departments like laboratory, CTs, MRI, X-rays and USG," advises Dr Banerji, Medical Director \& CEO, Woodlands Medical Centre, Kolkata (16). Waiting area should be common for these departments and should be equipped with electronic plasma screens displaying the services and facilities available in the college, Audio Visual Aids to enhance the oral health knowledge of the patients with a purpose to constructively utilize the patient's waiting time. It should have dental education posters giving information about various dental diseases and treatment option in local language to create patient awareness and knowledge. It would be highly appreciable to have a separate help desk for the enquiries of the patient. Certain features like smart cards for quick patient records retrieval, 'Express registration' interactive kiosks for patients to feed in their personal details may be used. "Interactive kiosks are actually great because the hospital will save on the reception or inquiry counter staff as many frequently asked questions will be answered by the machine. At Global Hospital, they have put up kiosks in some of the specialities," says Curian (16).

The academics details of the college like annual exams of the student, vacation should be displayed in the OPD notice board and website for better patient information. Student should maintain the records of their patients and should utilize emails, SMS facility or personal phone calls to inform their respective patients about change in any appointment date or time.

\subsection{Patient Attitude \& Educational Qualification}

Patient educational status, attitude and low priority accorded to oral health have a definite effect on the satisfaction and OPD of the dental college. Majority of the patient reporting or willing to undergo treatment at dental college belong to low socio-economic status who cannot afford costly dental treatment at private clinic. In a study conducted by Hayat $\mathrm{H}$ et al at Khyber College of Dentistry majority of the illiterate patients were highly dissatisfied with the dental treatment than patients belonging to higher educational status (17). Similar findings were reported by Patel $\mathbf{J}$ in a study conducted at Priyadarshini Dental College \& Hospital Thiruvallur Chennai (7). In a study conducted by Bhushan P et al $63.66 \%$ of the students interviewed were of the view that patient attitude was the main reason for low OPD (4). Due to non digitalization of patient records patients who loose registration slips, treatment card have to undergo the same complex process of OPD leading to delay in treatment. A patient should understand that oral health is as important as any other disease and that loss of records or not reporting on time for treatment leads to increase waiting time/appointment time which causes inconvenience to them only.

\section{Role of Behavior Change Communication}

Refers to such measures undertaken which when undertaken brings about behavioral changes among the population in seeking dental treatment. This task should be entrusted with the Public Health Department of the College.

Dental camps should be organized in consultation with the village panchayat, VHW and the ASHA worker. They should be asked to attend the screening camps. At the time of screening camps emphasis should be on motivating and increasing the awareness of dental disease rather than only on inspection and selecting cases for treatment. Posters about dental disease and treatment and general awareness should be in local language. The camps should be held as near to the village as possible so that the villagers are able to utilize the facilities provided at the camp. The students should take health talk preferably in local language with emphasis on need for regular oral health check up and treatment. It should be explained to the population that dental disease cannot be treated with the help of medicines alone; that they have to undergo treatment in order to be dentally fit. Efforts should be made to establish oral health camps at local PHC's along with medical camps

DOI: 10.9790/0853-150725964 $\quad$ www.iosrjournals.org $\quad 61 \mid$ Page


organized by government or on important health days like pulse polio programme for vide publicity and acceptance.

\section{Role of Health Workers}

College authorities should organize special awareness camp for the local PHC employees, village panchayats, Anganwadi workers, ASHA and village health worker regarding dental diseases, their effect on general health, their treatment and facilities available at the college. It is necessary to first create awareness among the health care workers who are in direct contact with the general population and to bring about behavioral changes among them.

ASHA and Village Health Worker should be educated about basic dental diseases and their treatment modalities so that they are able to guide the villagers appropriately. Oral health inspection of the eligible couples and pregnant mothers should be made mandatory and a column for this should be included in ANC card and ASHA, VHW should make sure that they undergo oral health check up. Children in the age group of 2 to 4 yrs and their parents should be explained about dental caries and its effect on mastication and general health. Children reporting for immunization should also undergo mandatory oral health check up.

\subsection{Dentist Behavior / Communication}

Mode of communication (language) between patient and dentist is also a major reason for dissatisfaction. The trainee doctors because of their academics pressure are always in a hurry and don't give enough time to explain the procedure to the patient. In a study conducted by Patel $\mathrm{J} 26 \%$ of the patient were dissatisfied with the communication or treatment procedure as explained by the dental students because the dentist did not know the local language (7). 53\% of the respondent said they had communication problem in a study conducted by Kashinath K R eta al (8). A survey in New Zealand (18) showed that almost all the patients wanted to be informed in detail about their dental treatment. Similarly, Rankin and Harris (19) reported that $97 \%$ of the patients surveyed favored a dentist who explained what the treatment and procedure entailed. To overcome this college should have dedicated interlocutors knowing local language to bridge the communication gap. They should explain in detail the dental diseases and treatment option to the patients if the students are not able to do so. Students reporting for their $1^{\text {st }}$ clinical duties should undergo special classes/lecturers regarding importance of behavior and communication with the patients. Students should be explained that patient work and satisfaction is equally important as academics and how to manage academics with patients work efficiently. They should focus on quality of treatment given rather than on quantity of patients they do.

\subsection{Distance}

$36.5 \%$ of the female respondent in Riyadh city said that the distance of the clinic from home is the most discouraging factor for non utilization of dental facility in a study conducted by Johara A, Hussyeen A (20) and $19.33 \%$ of the respondents were of similar views in a study conducted by Bhushan P et al (4). As most of the dental college \& hospital are situated on the out skirts of the city; distance can be regarded as a major reason for low OPD at the dental colleges. It should be seen in 3 aspects the actual distance a patient has to travel to reach the college, time taken to cover the distance and the mode of convenience.

\section{Dental Sub Centre}

To make the college services more accessible to population, the authorities should set up small dental centres like the PHC's located at important intersections of the city. These centres should have 3 to 4 dental chairs, 4-5 interns \& 2 to 3 para dental staff for patient work, teacher for supervision and a clerk for data entry on a rotational basis. These centres may provide basic OPD and treatment facilities whereas complicated cases may be referred to college hospital. These centres will not only reduce the travelling time but will also reduce treatment time for various basic treatments and increase the OPD of the college hospital significantly. The college authority may liaise with the government official and establish these subcentres along with the local PHC's and provide dental treatment free of cost to the patient reporting to these PHC's.

Also the college authorities may start dedicated patient bus service for people from far off places. Each bus should have a dedicated route and route chart and bus number should be displayed on the bus, college premises and also at important location of the city. Every day the bus should take the dedicated route and carry the patient to the college and also should drop them after a stipulated time at the designated bus stop or area.

\subsection{Example of Successful Use of IT}

Under Swiss Red Cross funding Electronic Patient Records (EPR) had been implemented in 27 government hospitals in the Eastern Province of Sri Lanka (21). Initially it was designed for Multi-Disease Surveillance project and the plan was to use computers mainly for recording in-patient details. However as the project proceeded, it became clear that major gains in efficiency and quality of documentation could be made by 
using computers in the OPD (21). Providing computers for the doctors to use in OPD has greatly increased the speed of documentation as well as providing access to the previous clinical record (21).

In one simple screen, the patient's personal details can be recorded at the front desk when the patient sees the doctor and the rest of the information can be entered in less than 30 seconds (data and time of the visit, staff involved, and patient complaint, treatment and lab tests) Fig 2.

The system also helps staff to order and visualize laboratory tests and x-rays. In the latter case, the computer can display a digital x-ray image on the screen thus reducing the costs of x-ray films (21). However the main benefit perceived by the medical staff is access to the previous medical record. On one screen, it is possible to see in overview the total record of the patient's attendances to the hospital - OPD consultations, Clinic visits and Admissions (21). Individual records can then be opened to show more detail (Fig 3).

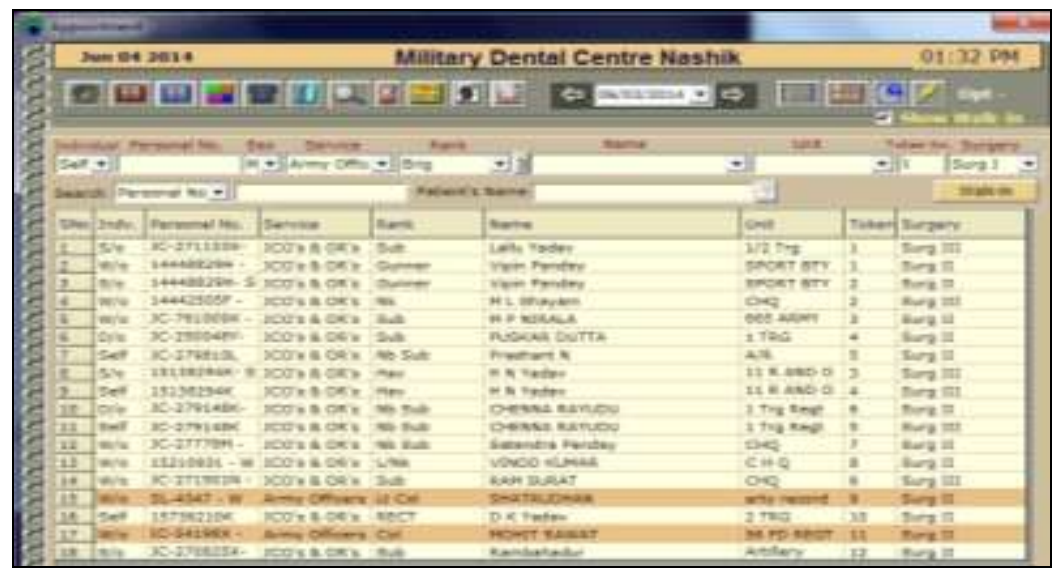

Fig 1 - Densoft Software at Reception of a Dental Centre (Indian Armed Forces)

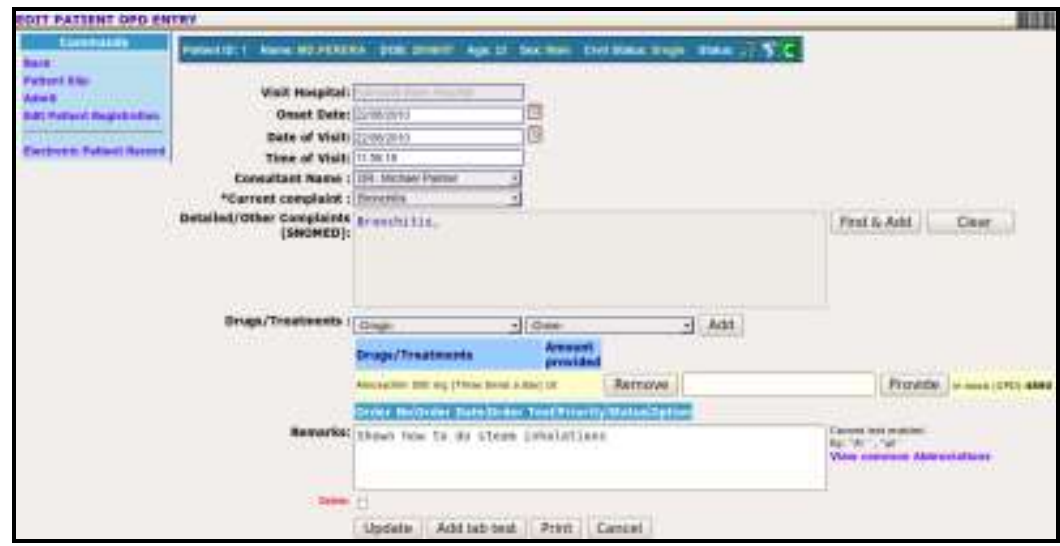

Fig 2 - Sample of Software to enter the out-patient record of the Patients

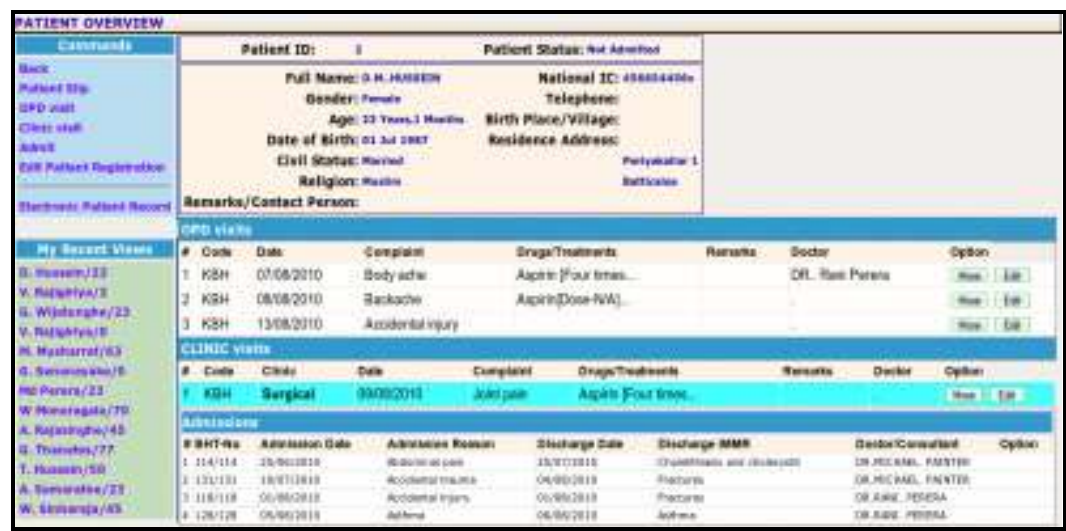

Fig3- Software Showing the Overview of the Entire Medical Record of Patient 


\section{Conclusion}

Despite improved dentist to population ratio and increased number of dental college still a lot of ground is to be covered to achieve oral health goals. Health for all by 2020 is incomplete without good oral health. There is urgent requirement of reorganizing the regional imbalance of dental colleges. Certain states and particular cities within that states have multiple dental college while the population of rest of the region of the same state have no access to the dental college and hospital.

There should be major shift or changes in the functioning of the OPD of the dental college. OPD should be arranged according to the needs of the patients and should not depend on the space available with the college authorities. College authorities should not wait for the patient to come to them but rather should reach out to the population and work on the upliftment of the oral health status of the population. Use of Information Technology in the OPD would immensely help in the smooth and efficient functioning of the OPD. Expenditure on IT would be one time however the benefits of this facility can be reaped for a long duration. Therefore implementation of IT in the OPD should be seriously thought about.

The current dental health programme emphasizes on curative aspect of dental diseases however the focus should shift to preventive aspect as most of the dental diseases are preventive in nature. Increased oral health awareness and social upliftment of population may cause significant improvement in the oral health status of the population.

\section{Reference}

[1]. Bali RK, Mathur VB, Talwar PP, Chanana HB. National Oral Health Survey and Fluoride Mapping 2002-2003 India. New Delhi: Dental Council of India in Collaboration with Ministry of Health and Family Welfare, Government of India; 2004.

[2]. Ferlay J, Soerjomataram I, Ervik M, Dikshit R, Eser S, Mathers C, Lyon, France: International Agency for Research on Cancer; 2013. Available from: http://www. globocan.iarc.fr/Pages/fact_sheets_population.aspx.

[3]. AhujaNK, Parmar R. Demographics and Current Scenario with Respect to Dentists, Dental Institutions and Dental Practices in India.Indian Journal Dental Sciences. 2011;3(2): 8-11.

[4]. Bhushan P, Kumar K, Prasanth MC. Study of Reason for Low OPD at SMBT Dental College: An Open Ended Questionnaire Survey. American Journal of Advances in Medical Science, 2014; 2(3): 26-33.

[5]. Dinesh TA, Singh S, Nair P, Remya TR. Reducing Waiting Time in Outpatient Services of Large University Teaching Hospital - A Six Sigma Approach. Manag Heal. 2013; 17(1): 23-34.

[6]. Hayat H, Rehman B, Din Q. Factors Affecting Patient Satisfaction and Delay in Urgent Care in Patients Reporting to Khyber college of Dentistry.Indian Journalof Dental Sciences. 2012; 3 (1): 22-27.

[7]. Patel JY. A Study on Evaluation of Patient Satisfaction with Dental Health Care Services.Int J Scie Res Pub. 2014; 4 (8): 1-4

[8]. Kashinath K R, Bharateesh J V, ChandanAgali, Mythri H, DarshanaBennadi, Mohan Kumar CT. Factors Affecting Patient Satisfaction among those Attending an Outpatient Department of a Dental College in Tumkur City - A Survey. Journal of Dental Sciences and Research. 2010;1(2): 1-10

[9]. Bither S, Gandhi S. An Audit of Patients Attending OPD of Oral and Maxillofacial Surgery at Christian Dental College, Ludhiana, Punjab, India.Journal of Education and Ethics in Dentistry. 2011; 1 (1): 28 -32

[10]. Densoft Dental Software.http://www.dentsoftltd.co.uk/

[11]. Dr SandeepMoolchandani. PPT on Hospital IT Department.www.slideshare.com

[12]. Wiley G. The PACS Payoff.Imaging Economics: The Journal of Imaging Technology Management.2003

[13]. Asghar S, Fatima F, Ali A, Amanat N. Assessment of Patient's Satisfaction about Dental Treatment/ Facilities Provided by Bahria Dental College Hospital, Karachi. Pakistan Oral \& Dental Journal. 2103; 33 (3): 535-538.

[14]. Taiwo O A, Soyele O O ,Ndubuizu G U . Pattern of Utilization of Dental Services at Federal Medical Centre, Katsina, Northwest Nigeria.Sahel Medical Journal.2014; 17 (3): 108-111.

[15]. Vashisth S, Gupta N, Bansal M, Rao N C. Utilization of Services Rendered in Dental Outreach Programs in Rural Areas of Haryana. ContempClin Dent. 2012; 3(2): 164-166.

[16]. Shukla S. Streamlining OPD. Insight into the Business of Healthcare.www.expresshealthcare.in. April 2008

[17]. Hayat H, Rehman B, Din Q U. Factors Affecting Patient Satisfaction and Delay in Urgent Care in Patients Reporting To Khyber College of Dentistry. JKCD. 2012; 3 (1): 22-27

[18]. Bishop TG, Flett RD, Beck DJ. A Survey of Dental Health Knowledge and Attitudes in Owaka, A Rural Community in South Otaga. NZ Dent J 1975; 71: 185-90.

[19]. Rankin JA, Harris MB. Patients' Preferences for Dentists' Behaviors. J Am Dent Assoc 1985; 110: 323-26.

[20]. Al Johara A. Al-Hussyeen. Factors Affecting Utilization of Dental Health Services and Satisfaction among Adolescent Females in Riyadh City.The Saudi Dental Journal.2010; 22; 19-25.

[21]. Pole D. Computerization of Clinical Records in Out-Patient Departments of Sri Lankan Hospitals. Sri Lanka Journal of Bio-Medical informatics. 2010;1(4):200-04. 